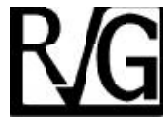

\title{
Implementación del enfoque de derechos humanos: la reforma sanitaria chilena*
}

\author{
Cunill-Grau, Nuria** \\ Fernández, Margarita ${ }^{\star \star \star}$
}

\section{Resumen}

El propósito de este trabajo es analizar la incidencia de la arquitectura institucional pública en la implementación de las políticas públicas con enfoque de derechos humanos. Se adopta como caso de estudio la reforma sanitaria chilena a partir del año 2005, que reconoce garantías exigibles de acceso, oportunidad, calidad y protección financiera para un conjunto de problemas de salud a la ciudadanía, independientemente de su adscripción al sistema público o privado. La metodología integró un análisis cualitativo de normas legales, instrumentos de gestión y entrevistas en profundidad principalmente a directivos de salud. Los resultados muestran que, no obstante los evidentes logros de la reforma chilena en pos de la equidad en salud, resultan limitados sus esfuerzos de dotar de gobernanza sistémica, accountability y espacio público a su arquitectura institucional, comprometiendo con ello su intención de instaurar un enfoque de derechos humanos. Se concluye que el patrón hegemónico de diseño institucional, organización y gestión de los servicios sociales requiere ser enfrentado para construir viabilidad a la implementación de este tipo de política y se sugiere la importancia de adoptar estrategias diferenciadas de accountabitily que consideren las asimetrías sociales en la apropiación y exigibilidad de los derechos.

Palabras clave: Derechos humanos, derechos a la salud, políticas públicas, gestión pública.

\section{Recibido: 20-09-12. Aceptado: 27-01-14}

* Este trabajo es una derivación del Proyecto FONDECYT N 1090433 ejecutado entre el 15/03/2009 al 15/03/2011 y titulado "La viabilidad de la institucionalidad pública para la ejecución de políticas con enfoque de derechos. Aproximación desde el caso de las reformas de la salud en Chile". Se agradece el financiamiento provisto por FONDECYT, CONICYT, Chile.

** Profesora-Investigadora del Centro de Investigaciones en Sociedad y Políticas Públicas (CISPO), Universidad de Los Lagos, campus Santiago y de la Escuela de Salud Pública, Universidad de Chile. E-mail: nuriacunill@vtr.net

*** Profesora-Investigadora del Centro de Investigaciones en Sociedad y Políticas Públicas (CISPO), Universidad de Los Lagos, campus Santiago. Universidad de Chile. E-mail: mmargaf@gmail.com 


\title{
Implementation of the Human Rights Approach: Chilean Health Reform
}

\begin{abstract}
The purpose of this work is to analyze the effect of public institutional architecture on implementing public policies with a focus on human rights. The Chilean health reform, from 2005 on, is adopted as a case study. It recognizes enforceable guarantees for citizens to access, opportunity, quality and financial protection for a set of health problems, independent of their subscription to the public or private system. The methodology integrated a qualitative analysis of legal norms, management instruments and in-depth interviews, principally with health directors. Results show that, despite the evident achievements of the Chilean reform for equity in health treatment, efforts to give systematic governance, accountability and public space to its institutional architecture are limited, thereby compromising the intention to install a human rights focus. Conclusions are that the hegemonic pattern of the institutional design, organization and management of the social services should be confronted in order to build viability into implementation of this type of policy. The study suggests the importance of adopting differentiated strategies for accountability that consider social asymmetries in the appropriation and enforceability of rights.
\end{abstract}

Key words: Human rights, rights to health, public policies, public management.

\section{Introducción}

Los derechos sociales suelen estar sometidos a la polémica acerca de si constituyen o no derechos fundamentales, con obligaciones y responsabilidades precisas para su protección. Sin embargo, esta polémica se dirime cuando una política social adopta el marco normativo de los derechos humanos aceptado internacionalmente. Así, la universalidad, exigibilidad, integralidad, participación social, y progresividad, vale decir los principios básicos de las Políticas Públicas con Enfoque de Derechos Humanos (PPEDH), pasan a iluminar el diseño de la política social en cuestión y a tener un respaldo legal (United Nations, 2002; Abramovich, 2006; Abramovich y Courtis, 2006; Pautassi, 2007; Agbodjan, 2005; Dworkin, 2002; Herreño, 2008).
Las políticas con enfoque de derechos humanos están ganando creciente terreno en América Latina y, con ellas, su estudio (Abramovich y Pautassi, 2009; Gherardi, 2009; Drago, 2006; Infante y Paraje, 2010; Pautassi, 2010; Erazo y Titelman, 2008; Notti, 2008; Canto, 2010). Sin embargo, poca atención se le ha brindado al conocimiento de los efectos que sobre una PPEDH tiene el tipo de diseño institucional y de patrón de gestión en los que se asienta así como sus traducciones en los valores y prácticas de los actores (Cunill, 2010).

El patrón guía, al menos desde la década de los 90, de la mayoría de las reformas institucionales de los servicios sociales (Banco Mundial, 1998 y 2004; BID, 1996), por su énfasis en el fomento de la competencia y, en general, por su adopción de la lógica de mercado, no tematiza sobre instrumentos y procesos de gestión en red que 
aumenten la percepción de la interdependencia (Christensen y Lægreid, 2007; Shankardass et al, 2012) y fomenten la ayuda mutua (Osborne, 2010; Agranoff, 2007; Cropper, et al 2008; Axelsson y Axelsson, 2006; Goldsmith y Eggers, 2006).

Por otra parte, el patrón hegemónico de reforma, al asentar el desarrollo de la accountability (responsabilización) básicamente en el aumento del "poder del cliente" sobre los prestadores de los servicios por medio de facilitar su "salida" y su "voz" individual, desdeña la importancia de arreglos institucionales traducidos en participación ciudadana deliberativa y autónoma en la formación, control y evaluación de las decisiones públicas (Fung y Wright, 2003; Fung, 2003; Santos y Avritzer, 2004; Walters et al, 2000), así como no se ocupa, más allá de la rendición de cuentas, de los requerimientos institucionales de la construcción de la accountability (Behn, 2001; Bourgon, 2007; Dowling et al, 2008).

Por tanto, puede conjeturarse que el patrón de reforma de los servicios públicos sociales que ha predominado en América Latina no es consistente con el tipo de arquitectura institucional requerida para la implementación de una PPEDH. De hecho, si se consideran los principios normativos de las mismas, es posible apreciar que ciertos atributos organizacionales son interpelados por ellos. El principio de la exigibilidad de los derechos, obliga a colocar la atención en la manera cómo se construye la accountability de los entes que resultan obligados.

El principio de la participación social, desde el punto de vista del diseño institucional, remite al grado en que las reglas de juego instituidas facilitan la construcción de un espacio público. Por su parte, el principio de la integralidad de los derechos remite a la medida en que el diseño institucional facilita una arquitectura colaborativa, o sea relaciones orgánicas y sostenidas de cooperación entre los actores involucrados en su despliegue, lo que acá se denomina gobernanza sistémica (Cunill et al, 2011; Cunill, 2010) ${ }^{1}$.

El artículo expone algunos de los resultados de una investigación realizada durante dos años a fin de comprender los nudos críticos que enfrentan esos tres atributos, accountability, espacio público y gobernanza sistémica, en el proceso de implementación de una PPEDH.

Para sustentar el análisis, se adopta como caso de estudio el de la reforma sanitaria chilena implementada a partir del año 2005 con base en la Ley de Autoridad Sanitaria (Ministerio de Salud, 2004a) y, más específicamente, la Ley de Acceso Universal con Garantías Explícitas en Salud -AUGE- (Ministerio de Salud, 2004b). Esta reforma, instalada en el

1 La expresión alude a las relaciones dentro de un sector gubernamental específico, compuesto a la vez de una variedad de actores que deben actuar como un todo para producir soluciones integrales a las problemáticas interpeladas por el derecho. Así planteada, la gobernanza es tanto efecto como resultado de "aquellos procesos colectivos formales o informales de toma de decisión y de elaboración de normas sociales con relación a asuntos públicos" (Hufty, 2010:5). 
marco de un sistema dual de salud ${ }^{2}$, crea obligaciones jurídicas (Garantías Exigibles de Derechos en Salud, GES) respecto de la oportunidad (tiempos de espera máximos), acceso (cobertura), protección financiera (tope máximo de pago anual por familia), y calidad (acreditación de las entidades y certificación de los médicos) del diagnóstico y tratamiento para un conjunto de problemas de salud ${ }^{3}$. La ley define las GES como dinámicas; deben actualizarse e incrementarse a través del tiempo bajo los parámetros y metodología establecidos en la propia ley (Drago, 2006:36), cuestión que efectivamente se ha hecho a través de sucesivos decretos, alcanzando 69 problemas de salud en el año 2012 (Ministerio de Salud, 2010). La priorización de los problemas ha tomado en cuenta, entre otros asuntos, prevalencia, equidad y vulnerabili- dad, así como capacidad de oferta y preferencias ciudadanas.

Las garantías son universales, o sea se encuentran disponibles tanto para los beneficiarios del seguro público como del privado. Así, la reforma sanitaria busca atenuar los efectos negativos de la dualidad del sistema chileno en la equidad y calidad en salud. Además, no circunscribe las garantías a prestaciones específicas sino a problemas de salud, abarcando desde la prevención hasta la rehabilitación. Por tanto, aunque en su origen no estuvo exenta de conflictos en torno a su alcance y a su financiamiento ${ }^{4}$, la reforma innova en cuanto a hacer exigibles por todas las personas las prestaciones asociadas a esos problemas de salud. Se asienta, pues, la noción de ciudadanos como titulares de derechos de salud (Infante y Paraje, 2010:83) que gene-

2 Este sistema es instituido durante el gobierno de Augusto Pinochet alcrearse aseguradoras privadas (Instituciones de Salud Previsional, ISAPRE) para la administración de la cotización obligatoria de los trabajadores (y vendieran seguros adicionales), compitiendo con el asegurador público (Fondo Nacional de Salud, FONASA). Este sistema dual se tradujo en que FONASA concentrara a las personas de menos ingreso y alto riesgo.

3 Los primeros problemas de salud reconocidos en enero del 2005 fueron 25 e incluían, entre otros, los siguientes (Ministerio de Salud, 2005a): Insuficiencia renal crónica terminal; Cáncer infantil, en menores de 15 años; Infarto agudo del miocardio; Cáncer cérvico-uterino; Cáncer de mama, en mayores de 15 años; Cáncer testicular, en mayores de 15 años; Linfoma en adultos; Diabetes Mellitus, tipo I y tipo II; Esquizofrenia.

4 En el gobierno habían dos posiciones: una sostenía la necesidad de aumentar el gasto fiscal en salud y otra asumió el enfoque de derechos aunque solo con relación a un conjunto de problemas de salud (que prevaleció). Por otra parte, hubo una activa discusión acerca de la modalidad de financiamiento. La propuesta original del gobierno (posteriormente retirada, dada la oposición de las ISAPREs asumida por los parlamentarios de oposición) era crear un fondo de compensación de riesgos entre las ISAPREs y FONASA para disminuir la segmentación entre seguros de salud y aumentar la equidad en todas las patologías incluyendo las no garantizadas explícitamente. Al final se creó fue un Fondo INTER-ISAPREs. Luego de fracasar la idea original para financiar la reforma en el sistema público, se propuso recurrir a varios impuestos (tabaco, alcoholes, entre otros) pero lo aprobado fue un aumento en el Impuesto al Valor Agregado (IVA). La discusión parlamentaria se extendió por dos años (Infante y Paraje, 2010). 
ran obligaciones para otros, enmarcándose dentro del enfoque de los derechos humanos (Drago, 2006:8). La reforma se propone además, entre otros asuntos, fortalecer las redes asistenciales con énfasis en la atención primaria, teniendo en vista un modelo preventivo y con foco en la familia.

Este estudio, de tipo cualitativo, transeccional y multimétodo, indagó acerca de las dimensiones de gobernanza sistémica, accountability, y espacio público en el proceso de implementación de la reforma (2005-2010) en el sistema público de salud. En este contexto, el sistema público es considerado el soporte principal de la reforma mientras que el sector privado solo opera bajo relaciones de compra-venta de servicios tanto entre sí (compra) como con el sector público (básicamente venta).

Se contempló: a) un análisis de contenido del marco legal-reglamentario y de la documentación oficial de la reforma, a fin de establecer cómo se aborda la construcción de gobernanza sistémica, accountability y espacio público; b) entrevistas en profundidad a una muestra de directivos de toda la red asistencial seleccionados en forma trietápica, orientadas a indagar sus percepciones sobre los nudos críticos y significados que le asignan a la gobernanza, espacio público y accountability.

Entre noviembre y diciembre de 2009 , se entrevistaron 40 directivos de di- ferentes tipos de establecimientos asistenciales bajo la jurisdicción de cinco Servicios de Salud (SS) ${ }^{5}$; éstos fueron seleccionados al azar dentro de tres regiones elegidas por su mayor concentración poblacional, dos urbanas y una rural (Región Metropolitana, Región del Libertador Bernardo O'Higgins, y Región de BíoBío). Se incluyó también a niveles directivos superiores (Subsecretario de Redes Asistenciales y Superintendente de Salud). Posteriormente, entre septiembre y noviembre de 2010 , se realizaron 10 entrevistas individuales a los encargados de participación de los SS, así como 14 entrevistas grupales a 20 encargados de la red territorial de participación y a 31 dirigentes sociales.

En el territorio de la jurisdicción de cada SS, se entrevistaron a directivos de las redes asistenciales que los SS articulan; en particular, de un hospital de alta complejidad y otro de media complejidad; de dos Corporaciones o Departamentos de Salud Municipales, una correspondiente a una comuna con mayor concentración urbana y la otra correspondiente a una comuna definida como vulnerable por el Ministerio de Salud y dentro de ellas, al azar, de un Centro de Salud Familiar (CESFAM) 6 .

Los entrevistados manifestaron su consentimiento informado bajo condiciones de confidencialidad, el que fue validado previamente por la Comisión Ética de SS O’Higgins.

6 Los CESFAM son centros de atención ambulatoria del primer nivel definidos para operar bajo un modelo de salud familiar. 
la Facultad de Ciencias de la Universidad de Chile y por el Comité Asesor de Bioética del Fondo Nacional de Desarrollo Científico y Tecnológico (FONDECYT). Los resultados del análisis documental y de las entrevistas fueron triangulados y analizados con el programa Atlast1.

\section{Hallazgos sobre la gobernanza sistémica del sistema público}

Los cambios institucionales buscaron plasmar el enfoque de derechos en la reforma sanitaria chilena e incluyeron el refuerzo de las funciones de rectoría, configurando la autoridad sanitaria a nivel nacional y regional, para lo cual se entregaron nuevas atribuciones al Ministerio de Salud y a las secretarías regionales ministeriales (Ministerio de Salud, 2004a). También se buscó fortalecer la gestión de la red asistencial, separándola de las funciones de salud pública y asignando al director de cada SS el rol de gestor de red y de encargado de un Consejo Integrado de la Red Asistencial (CIRA). Las funciones de regulación se tradujeron en nuevas atribuciones de la Superintendencia de Salud, encargándole la fiscalización del cumplimiento de las GES en el sistema público.

A estos cambios, se sumó la readecuación de la atención primaria hacia un enfoque de salud familiar. Finalmente, se buscó el alineamiento de los actores del sistema público en torno al cumplimiento de los derechos garantizados a través de las propias GES y de las Metas Sanitarias dictadas por decretos con fuerza de ley, por tanto de obligatorio cumplimiento, y a las que se asociaron incentivos económicos (Ministerio de Salud, 2006).
Así, los cambios incorporados por la reforma implicaron tanto la aparición de nuevos actores como la necesidad de configurar sistemas de relación entre ellos, para responder a los desafíos del enfoque de derechos, en particular al desafío de la integralidad.

Ahora bien, el análisis del marco legal-reglamentario y de la documentación oficial de la reforma (Ministerio de Salud, 2004a, 2004b, 2004c, 2005b y 2006) realizado para contrastar los requerimientos relativos a la producción de gobernanza sistémica con la institucionalidad construida, releva tres ámbitos a los cuales la reforma prestó una débil atención: a) la creación de instancias para fomentar la gestión en red, por cuanto la única instancia, el CIRA, tiene sólo carácter consultivo $y$ funciones poco definidas; b) el establecimiento de metas que estimulen la gestión en red; y c) la existencia de instrumentos de planificación, presupuesto y evaluación conjuntas.

Por su parte, las entrevistas a los actores tienden a confirmar sesgos en cada una de esas tres dimensiones. De hecho, los cinco directores de SS entrevistados (Entrevistado 7, Entrevista 2009; Entrevistado 1, Entrevista 2009; Entrevistado 16, Entrevista 2009; Entrevistado 18, Entrevista 2009; Entrevistado 2, Entrevista 2009) coinciden en que la coordinación de la red asistencial, pilar de una gestión con foco en el cumplimiento de los derechos, presenta vacíos. Por lo tanto, el funcionamiento de las instancias de articulación, en especial de los CIRA, es altamente dependiente de la voluntad de los directivos de los diversos niveles de atención.

Algunos de esos directores (Entrevistado 18, Entrevista 2009; Entrevistado 
16, Entrevista 2009) señalan, que aun cuando la diferenciación de funciones entre prestación asistencial y autoridad sanitaria permite "concentrarse mejor" en cada una, ha debilitado el funcionamiento del sistema público de salud por la ausencia de arreglos institucionales para vincular los temas de salud colectiva con los servicios de atención asistencial de las personas bajo un enfoque integral. El principal argumento al respecto, es que las fragilidades de los mecanismos de diálogo y coordinación impiden a los SS y a la propia Autoridad Sanitaria regional compartir diagnósticos, visiones y propuestas sobre las problemáticas de salud y medioambiente que afectan en un determinado territorio.

Por otra parte, según las percepciones de algunos de los directores y subdirectores de los SS (Entrevistado 1, Entrevista 2009; Entrevistado 2, Entrevistas 2009, Entrevistado 7, Entrevistas 2009; Entrevistado 16, Entrevista 2009; Entrevistado 12, Entrevista 2009; Entrevistado 8, Entrevista 2009; Entrevistado 17, Entrevista 2009) y de directores de los hospitales (Entrevistado 9, Entrevista 2009; Entrevistado 11, Entrevista 2009; Entrevistado 19, Entrevista 2009; Entrevistado 13, Entrevista 2009), el cumplimiento de las prestaciones tampoco deja espacio para la adaptación de las políticas a cada realidad regional. Además, destaca el hecho de que las metas, al responder a lógicas provenientes del Ministerio de Hacienda, no estimulan la cooperación y ayuda intra sistema e, indirectamente, fomentan la contratación de servicios al sistema privado para darles cumplimiento.
De los Centros de Salud Familiar (Entrevistado 15, Entrevista 2009; Entrevistado 10, Entrevista 2009; Entrevistado 6 , Entrevista 2009) aflora el hallazgo de que el esfuerzo de construcción de este modelo ha tendido a ser desplazado por los objetivos transversales de la reforma (GES, Metas Sanitarias) y por los instrumentos de gestión planificados centralmente, quienes estructuran de hecho la acción de los equipos, retrotrayéndola a la concepción biomédica en vez de permitirle avanzar a una concepción bio-psico social de la salud. El problema central, en este sentido, lo sintetiza uno de los directores entrevistados (Entrevistado 15, Entrevista 2009): "Creo que la reforma no ha explicitado para dónde quiere ir en la Atención Primaria de Salud".

El análisis de las entrevistas efectuadas a diez directores de las corporaciones o departamentos de salud de los municipios (Entrevistado 4, Entrevista 2009; Entrevistado 5, Entrevista 2009; Entrevistado 14, Entrevista 2009; Entrevistado 21, Entrevista 2009; Entrevistado 22, Entrevista 2009; Entrevistado 23, Entrevista 2009; Entrevistado 24, Entrevista 2009; Entrevistado 25, Entrevista 2009; Entrevistado 26, Entrevista 2009; Entrevistado 27, Entrevista 2009) sugiere como mayor nudo crítico el excesivo centralismo en la elaboración de las normas y en la definición de los instrumentos de gestión. En general, los entrevistados sostienen que se requeriría de un flujo bidireccional capaz de considerar las brechas y la epidemiología local, los procesos de reconversión de la salud en salud familiar, las realidades geográficas y las limitaciones de recursos de las municipalidades. 
Por otra parte, recurrentemente aflora la percepción de que la gobernanza del sector salud en los territorios se complejiza aún más porque está mediada por la voluntad política de los alcaldes, quienes convertidos en actores clave en el proceso de municipalización de la salud primaria en los 80 , inciden diferenciadamente en la inversión en salud local.

Finalmente, el estudio muestra las relaciones intersectoriales como uno de los asuntos menos atendidos por el proceso de reforma, a pesar de que de ellas depende en gran medida la eficacia de las acciones de prevención y protección de la salud. Además, el tema de la intersectorialidad prácticamente está ausente del discurso de los entrevistados.

\section{Nudos críticos de la accountability (responsabilización)}

Una de las conclusiones derivadas del análisis documental es que la reforma sanitaria en el campo de la accountability desarrolla una variada gama de órganos y recursos para la exigibilidad de los derechos y la resolución de conflictos que trascienden, aunque también incluyen, los típicos derivados de las leyes de procedimientos administrativos y del poder judicial.

Primero, el marco normativo de la reforma (Ministerio de Salud, 2004a) dispone la obligación de los prestadores de salud de informar y señala mecanismos específicos de información sobre los de- rechos y vías para reclamar en caso de incumplimiento. Contempla también una institucionalidad independiente para asegurar la exigibilidad de los derechos, la Superintendencia de Salud, tanto en el sector público como en el sector privado.

Segundo, el mismo marco de la reforma (Ministerio de Salud, 2004a) abre la posibilidad de la mediación pre-judicial de parte de la Superintendencia de Salud (para los asegurados del sistema privado) y del Consejo de Defensa del Estado (para los asegurados en el sistema público). Asimismo, activa recursos administrativos que pueden ser interpuestos ante las propias aseguradoras y plazos específicos para hacer valer las garantías de acceso y oportunidad. Por lo tanto, pone especial atención en generar instancias previas a la judicial, cumplidas básicamente por la Superintendencia de Salud. Con ello, se manifiesta la preocupación de la reforma sanitaria por diversificar los recursos directos a los que puede recurrir la ciudadanía.

Con respecto al cumplimiento de la garantía de calidad, desarrolla varios instrumentos entre los que destaca un sistema de acreditación. Este último se construye a partir de la creación de una Intendencia de Prestadores en la Superintendencia de Salud, la definición de estándares de calidad (protocolos y guías clínicas), y la contratación de entidades acreditadoras (Ministerio de Salud, 2005c, 2007).

Con relación a la rendición de cuentas, se recoge la indicación del gobierno de la presidenta Michelle Bachelet 
(División de Organizaciones Sociales, 2009) ${ }^{7}$ de asumir la rendición de cuentas de todas las autoridades como un proceso que implica la constitución de un espacio deliberativo, más no resolutivo, con representantes de la sociedad civil respecto del desempeño y la acción de cada servicio público.

Sin embargo, el estudio sugiere que existen varios obstáculos al uso de los mecanismos de exigibilidad. Uno clave atinge a la información sobre los derechos. Todos los entrevistados llaman la atención acerca de la complejidad del contenido de la información a entregar a los usuarios, las dificultades de los propios establecimientos para apropiarse de la información, la ausencia de políticas diferenciadas de información para usuarios urbanos o rurales y con distintos niveles educacionales, y la escasa difusión de los deberes ciudadanos. También se observan tensiones respecto a quién y cómo corresponde informar. El rol de los médicos como fuentes esenciales de información aparece permanentemente relevado, pero también el hecho de que no siempre es ejercitado tanto por resistencias a aumentar la presión sobre ellos, como por los déficits de los sistemas de información disponibles en el sector.

Otro nudo crítico concierne a los variados obstáculos al uso de los procedimientos de reclamo. Así, la exigibilidad está asociada a la formalización del reclamo (limitado a 3 días y recién extendido a 15 días), sin el cual no es posible activar el sistema de garantías. Por otro lado, los asegurados por el sistema público, al estar obligados a acudir al primer nivel de atención para hacer valer sus garantías, deben acogerse al régimen jurídico de los establecimientos del nivel municipal. Si la municipalidad ha creado una corporación de derecho privado para administrar la atención primaria, los perjuicios sufridos por el usuario no pueden ser dirimidos a través de las mediaciones realizadas a través del Consejo de Defensa del Estado (que opera para el sector público) y, por ende, no pueden ser gratuitas. Esto inhibe no solo el uso de las mediaciones sino el acceso a la justicia, tal como lo evidencian los datos disponibles respecto de ambos (Cunill, 2011).

Un tercer nudo crítico es la presencia de diferencias en la exigibilidad de las propias garantías, en contra de la garantía de calidad. Conceptualmente, la acreditación debe medir que las instituciones hayan definido y apliquen procesos de mejora continua de la calidad en los aspectos críticos para la seguridad de los pacientes. Sin embargo, aún no se generan mecanismos de sanción para exigir la acreditación. Por otra parte, los Protocolos y Guías Clínicas, considerados en la ley (Ministerio de Salud, 2005c, 2007), como el otro instrumento para garantizar la Calidad, no son de uso obligatorio.

Todo lo anterior, más el predominio de contradicciones entre las distintas agencias de la ciudadanía, sugiere que el sistema institucional de exigibilidad del 
sector público es débil. Con relación a esto el $90 \%$ de los 31 dirigentes sociales entrevistados en el año 2010 que intervenían en las instancias de participación establecidas, percibe la rendición de cuentas de los órganos de las redes asistenciales como una formalidad y no como un ejercicio real de accountability.

\section{Hallazgos sobre el espacio público construido por la reforma}

La reforma buscó también fomentar la participación ciudadana. De hecho, el discurso presidencial de Ricardo Lagos del año 2002 (Cámara de Diputados, 2002), en alusión al proyecto de ley que establece el régimen de garantías en salud, revela aspectos fundamentales a considerar con respecto a la relación con la ciudadanía como son: la rendición de cuentas sobre la calidad, efectividad y oportunidad de servicio; la adecuada y transparente información a la misma para una participación informada; y la apertura de espacios y canales a través de los cuales pudieran expresar sus preferencias.

Sin embargo, cuando la ley en referencia fue aprobada (Ministerio de Salud, 2004b) sancionó las GES dejando en manos de un Consejo consultivo ministerial, constituido por ciudadanía experta y del propio Ministerio, la definición de los derechos. Por otra parte, si bien fueron ampliadas las esferas de participación contempladas en la Ley de Autoridad Sanitaria (Ministerio de Salud, 2004a), establecieron el predominio de la participación de los estamentos técnicos y trabajadores. Con respecto a los usuarios solo se contempló su participación en los Conse- jos Consultivos de Hospitales que pasarían a ser auto-gestionados.

En el año 2006, el planteamiento presidencial del "Gobierno Ciudadano", contribuye a producir en el país una Agenda Pro-Participación (Ministerio Secretaría General de Gobierno, 2007) la cual, a pesar de ser recortada en la Ley № 20.500 (Ministerio Secretaría General de Gobierno, 2011), establece un "piso mínimo" a cumplir: el derecho a la información y la queja a través de la instalación de las Oficinas de Información, Reclamos y Sugerencias (OIRS). También contempla la formalización de Consejos Consultivos en los establecimientos y la incorporación de los usuarios al CIRA, para promover su voz en las redes asistenciales. Esta política se incorpora en los instrumentos de gestión del período 2005-2008, incluyendo metas pro-participación en las Metas Sanitarias (de cumplimiento obligatorio, como ya se ha señalado) y en los Compromisos de Gestión, los que son elegibles por los SS (Ministerio de Salud, 2008a).

El balance efectuado por el Ministerio de Salud al 2009 da cuenta de resultados positivos en la instalación de las OIRS $(96 \%$ de los establecimientos de salud), celebración de cuentas públicas (100\% de los SS y $68 \%$ de los hospitales) y creación de los Consejos Consultivos ( $81 \%$ de los establecimientos y el $100 \%$ de los SS) (Ministerio de Salud, 2009). Para los actores directivos entrevistados este salto se explica por su integración a las metas sanitarias en el año 2008, las cuales conllevan un incentivo monetario de desempeño.

Por otra parte, en los años 2008 y 2009 se instalan presupuestos participa- 
tivos que involucran a usuarios de 140 comunas de las 340 existentes (Pozo y Cancino, 2010). En cuatro SS se desarroIlan estos procesos en alianza con las Secretarías Regionales Ministeriales para priorizar áreas del Plan de Salud Pública 2006-2010 (Ministerio de Salud, 2008b) y en una de las regiones en alianza con el organismo asegurador público, quien los utiliza para priorizar atenciones a patologías no integradas en las GES, las cuales cuentan con largas listas de espera.

A pesar de la aplicación de esta política, los SS difieren sustantivamente en la amplitud y calidad de las estrategias de participación implementadas. De los cinco servicios estudiados, solo dos habían configurado al 2010 estrategias pro-participación a través de mecanismos de participación complementarios entre sí: consejos de usuarios, diálogos ciudadanos, presupuestos participativos, cartas de derechos, comités de solicitudes ciudadanas, integración de los usuarios al CIRA, cuenta pública. En estos servicios, destacan tres aspectos que actúan como condiciones facilitadoras: a) la voluntad directiva para impulsar espacios de participación inclusivos y deliberantes; b) la construcción de soportes institucionales de impulso a la participación, incluyendo la creación de departamentos de participación dotados de recursos profesionales; y c) la configuración de redes de encargados de participación en el territorio para adaptar las estrategias al medio.

Con respecto a la calidad de estos espacios de participación, las entrevistas a los encargados de participación y a dirigentes sociales (Entrevista Grupal 1, Entrevista 2010; Entrevista Grupal 2, Entrevista 2010; Entrevista Grupal 3, Entrevis- ta 2010; Entrevista Grupal 4, Entrevista 2010; Entrevista Grupal 5, Entrevista 2010) dan cuenta de un mejor funcionamiento en los SS donde los territorios son pequeños, urbanos, y con redes sociales densas y de larga data. En tales servicios, los espacios de participación tienden a ser deliberantes e incidentes en decisiones, aunque en ellos participan pequeños grupos sociales no articulados en redes y con una agenda restringida a las inversiones y al logro de las metas sanitarias.

Según los hallazgos de las entrevistas, para la mayoría de los directivos de Servicios y de Salud Municipal (Entrevistado 1, Entrevista 2009; Entrevistado 2, Entrevista 2009; Entrevistado 21, Entrevista 2009; Entrevistado 14, Entrevista 2009; Entrevistado 22, Entrevista 2009; Entrevistado 16, Entrevista 2009; Entrevistado 4, Entrevista 2009; Entrevistado 5, Entrevista 2009) la perspectiva de derechos no irradia a la participación social.

Por esta razón, salvo excepciones, los esfuerzos no logran influir en la creación de un espacio público que vincule la agenda de participación con la de los derechos. Conspiran contra ello, la falta de autonomía de los actores sociales, la baja representatividad de los líderes, su escasa rotación, así como la centralización de las decisiones, la que reduce la agenda de diálogo a las materias sobre las cuales los servicios tienen atribuciones directas.

En todo caso, uno de los hallazgos más importantes es que los arreglos institucionales de participación son influenciados por los sentidos otorgados por los directivos de los SS. Se puede reconocer en ellos cuatro perspectivas: a) de democratización de las decisiones de salud, asignando a la participación una impor- 
tancia estratégica en la construcción de equidad, plasmada en arreglos institucionales amplios de participación, sea a través de innovaciones o de adaptaciones de la oferta central; b) una instrumental, que asigna importancia a que la oferta pública sea pertinente a las necesidades de los usuarios, lo que supone abrir solo espacios de escucha; c) otra perspectiva más tecnocrática, limitada a instalar formalmente los dispositivos impuestos desde fuera; d) por último, una perspectiva de desencanto, que reconoce la importancia de la participación social mas no la estimula, considerando la baja formación de capital ciudadano y el predominio del clientelismo.

\section{Panorama global: diseño institucional con inconsistencias frente a los principios normativos de una PPEDH}

Los resultados de la investigación acerca de la gobernanza sistemática, la accountability y el espacio público creados en la institucionalidad chilena a partir de la reforma sanitaria sugieren que no se problematizó suficientemente acerca del tipo de adaptación requerida por las reglas, los modos de gestión y de organización vigentes, así como tampoco el tipo de relaciones que era necesario construir con la ciudadanía, afectando negativamente con ello la integralidad de los derechos, su exigibilidad y la participación social.

Específicamente, con relación a la gobernanza sistémica, los resultados insinúan que la lógica de los instrumentos de gestión basados en resultados, que replican los vigentes en todo el sector públi- co chileno, no contribuye a construir relaciones orgánicas para el manejo de una PPEDH sino más bien fomentan la competencia entre los actores 0 , en su defecto, el desarrollo de redes coyunturales y la recurrencia al sector privado, para la obtención de los incentivos económicos asociados al desempeño (Cunill et al, 2011).

Probablemente, la mejor justificación de la necesidad de gobernanza que conlleva la reforma queda reflejada en el siguiente aserto de uno de los directores entrevistados (Entrevistado 3, Entrevista 2009): "Antes nosotros estábamos más orientados hacia la administración de los hospitales. Ahora, debemos coordinar, aunar esfuerzos en torno a la salud".

Las limitaciones de recursos del sector obstaculizan este trabajo pero también, según los diversos hallazgos, la existencia de una institucionalidad que no favorece suficientemente la integración a nivel territorial, así como entre las funciones asistenciales y de promoción, y dificulta el cambio de modelo de atención dado su excesivo énfasis en metas productivistas asociadas a beneficios económicos.

Con respecto al desarrollo de la accountability, los resultados muestran déficits asociados al diseño mismo del sistema institucional de exigibilidad; éstos limitan especialmente los derechos de la población atendida por el sistema público, y en especial a los pobres. En efecto, uno de los asuntos particularmente crítico, que afecta directamente la equidad, es la capacidad diferencial de la población para asegurar la exigibilidad de las GES según su nivel socioeconómico. Influye en ello fundamentalmente el hecho de que la información sobre los dere- 
chos (incluido el conocimiento de la existencia de la Superintendencia de Salud) no ha tomado en consideración, ni en sus formatos ni en sus formas de acceso, la distribución desigual de la capacidad de uso de la información.

Refrendan las asimetrías sociales en el uso del sistema de exigibilidad de las garantías en salud el que los procedimientos de reclamo, por la manera en la cual han sido diseñados, sirven más a los asegurados del sistema privado que del público. La institucionalidad fiscalizadora de las obligaciones del sector salud tiene también limitaciones. De hecho, uno de los principales problemas percibidos por el Superintendente de Salud (Entrevistado 20, Entrevista 2009) es la adscripción de la Superintendencia de Salud al propio Ministerio de Salud, afectando su autonomía. Por otra parte, las facultades en términos de sanciones y multas no son equivalentes en el sector público comparadas con las del sector privado, lo cual limita la fiscalización sobre el primero.

La paradoja es que la igualación de garantías para un conjunto de patologías, las GES, atendidas ya sea por prestadores públicos o privados, no resulta suficiente para igualar las oportunidades sociales de disfrute de los derechos en salud. Esto, habida cuenta de que el propio sistema institucional de exigibilidad mantiene diferencias, de hecho y de derecho, en contra de los usuarios del sector público y en especial de los de menores recursos.

En lo concerniente al espacio público, los resultados muestran que los espacios de participación incluidos en los marcos legales de la reforma resultaron restrictivos respecto de la intencionalidad planteada en sus comienzos. La asociación de los espacios de participación a metas de desempeño ha tendido al vaciamiento de sus contenidos, transformándolos básicamente en "obligaciones a cumplir" para lograr compensaciones económicas. Por otra parte, el énfasis está puesto en la relación con los usuarios en tanto clientes y no con la ciudadanía, lo cual explica la importancia concedida a la institucionalización de oficinas de quejas y sugerencias y de órganos consultivos.

Por su parte, la política de participación del sector, aunque va ampliando los mecanismos en el período, debido a cambios en la agenda gubernamental, se orienta específicamente hacia las comunidades locales usuarias del sistema público, ámbito desde el cual, dado el centralismo prevaleciente en la toma de decisiones, se dificulta la incidencia en derechos.

Destaca además que la naturaleza de los espacios de participación ha sido dependiente de los sentidos otorgados por los directivos de los Servicios de Salud, los cuales se traducen en diferencias respecto de los soportes institucionales construidos para su impulso, los roles asignados a la ciudadanía, y los mecanismos utilizados para su incidencia en decisiones. Pero, aun en aquellos Servicios de Salud donde los directivos otorgan a la participación ciudadana un sentido democratizador, operan restricciones contextuales expresadas sobre todo en debilidades en la representatividad y autonomía sociales, todo lo cual limita la democratización efectiva de dichos espacios y su transformación en un espacio público. 


\section{Conclusiones}

Aun cuando la multiplicidad de factores en juego no permite establecer relaciones de causalidad absolutas, el estudio muestra que cómo el diseño de la institucionalidad encargada de la implementación de una PPEDH afecta su capacidad de realizar los derechos consagrados jurídicamente.

Tanto el análisis de las configuraciones institucionales, como de la percepción que tienen acerca de ellas los directivos de los distintos niveles de las redes asistenciales que conforman el sistema público de salud en Chile, sugieren que cuando no es problematizado el patrón hegemónico de diseño institucional, organización y gestión de los servicios sociales, no se logra construir plenamente gobernanza sistémica, ni condiciones para la operación de un espacio público que creen viabilidad institucional a la integralidad y a la participación social, consustanciales al marco normativo de un enfoque de derechos humanos.

En este sentido, los hallazgos muestran que la gestión en red no es suficiente para facilitar la colaboración y la comprensión mutua de las interdependencias entre los diversos actores y la adaptabilidad a las condiciones locales, cuando los compromisos de desempeño, las condiciones de su cumplimiento y de su evaluación se definen unilateralmente, crean incentivos para la competencia, y no dan cuenta de la necesidad de trabajo conjunto tanto dentro del sector salud como intersectorialmente.

También los resultados del estudio muestran que cuando no se brinda aten- ción expresa a la institucionalización de espacios deliberativos, autónomos y representativos donde la ciudadanía pueda incidir en las decisiones públicas y ejercer una activa vigilancia sobre sus resultados, los espacios de participación se hacen dependientes de la voluntad política de las autoridades o bien su legitimidad queda en entredicho. Cabe además considerar el riesgo de formalización de los espacios e instancias cuando son asociados a compromisos de gestión vinculados a beneficios económicos.

Finalmente, el aspecto más sensible de una PPEDH, que es el aseguramiento de la exigibilidad de los derechos por parte de la ciudadanía, según lo sugiere el estudio, no solo supone crear las condiciones institucionales de accountability de los órganos portadores de obligaciones, sino que además requiere de estrategias diferenciadas para mitigar las asimetrías sociales especialmente las relacionadas con el acceso y uso de la información sobre derechos; la disponibilidad de recursos administrativos y judiciales expeditos; y agencias estatales independientes que puedan actuar sinérgicamente en representación de la ciudadanía para la defensa de sus derechos.

Como lo muestra el caso chileno, estos asuntos son particularmente críticos cuando las garantías en salud operan tanto para los asegurados en el sistema público como en el privado, habida cuenta del riesgo de que los recursos de exigibilidad no puedan ser efectivamente utilizados por el sistema público y en especial por los pobres, contraviniendo así el enfoque de derechos humanos que se intentan consagrar. 
En suma, si bien el contenido de una PPEDH y su debido financiamiento son clave para la efectiva ampliación de los derechos sociales, queda sugerido a través de este estudio que, para allanar efectivamente el camino de la construcción de ciudadanía social, además sería necesario prestar atención al tipo de institucionalidad que en el campo público puede construirle viabilidad.

\section{Referencias Bibliográficas}

Abramovich, Víctor (2006). Una aproximación al enfoque de derechos en las estrategias y políticas de desarrollo. Revista de la CEPAL, abril, 88, pp 35-50.

Abramovich Víctor, y Courtis, Christian (2006). El umbral de la ciudadanía. El significado de los derechos sociales en el Estado social constitucional. Buenos Aires: Ediciones del Puerto.

Abramovich, Victor y Pautassi, Laura (2009). El enfoque de derechos y la institucionalidad de las políticas sociales. En: Abramovich, Victor, Pautassi, Laura (comps.). La revisión judicial de las políticas sociales. Buenos Aires: Ediciones del Puerto.

Agbodjan, Didier (2005). Las instituciones en pro de la exigibilidad y la justiciabilidad de los Derechos Económicos, Sociales y Culturales. En: Canto Chac, Manuel (editor), Derechos de ciudadanía: responsabilidad del Estado. México: Icaria.

Agranoff, Robert (2007). Managing within Network: Adding Value to Public Organizations, Washington, Georgetown University Press.

Axelsson Runo y Axelsson Susanna (2006). Integration and collaboration in public health. A conceptual framework. International Journal of Health Planning and Management, 21(1), pp. 75-88.
Banco Mundial (1998). Más allá del Consenso de Washington: la hora de la reforma institucional. Washington: Banco Mundial.

Banco Mundial (2004). Servicios para los pobres. Informe sobre el desarrollo mundial 2004. Washington: Banco Mundial.

Behn, Richard D. (2001). Rethinking Democratic Accountability. Washington: Brookings Institution Press.

BID (1996). Progreso económico y social en América Latina: cómo organizar con éxito los servicios sociales. Washington: BID.

Bourgon, Joceline (2007). Gobierno receptivo, responsable y respetado. Hacia una "nueva" teoría de la Administración Pública. Revista Internacional de Ciencias Administrativas, 73(1), pp. 5-26.

Canto Chac, Manuel (2010). Políticas públicas y derechos humanos. En: Estévez Ariadna y Vásquez Daniel (coord.) Los derechos humanos en las ciencias sociales: una perspectiva multidisciplinaria. México: Flacso México-Cisan.

Cámara de Diputados (2002). Mensaje al país del Presidente de la República Ricardo Lagos Escobar, 21 de mayo de 2002. Santiago, Chile. En: http://www.camaradediputados.cl/camara/media/docs/discursos/21mayo_2002.pdf (fecha de consulta: 18 de junio de 2009).

Christensen, Tom y Lægreid, Per (2007). Reformas post Nueva Gestión Pública: Tendencias empíricas y retos académicos. Gestion y Política Pública, CIDE, México, 16(2), pp. 539-564.

Cropper, Steve; Ebers, Mark; Huxshamn, Chris y Smith Ring, Peter (edits.) (2008). Introducing Inter-organizational Relations. En: Cropper, Steve; Ebers, Mark; Huxham, Chris; Smith Ring, Peter (edits.), Interorganizational relations. UK. Oxford: University Press. 
Cunill Grau, Nuria (2010). Las políticas con enfoque de derechos y su incidencia en la institucionalidad pública. Revista del CLAD Reforma y Democracia, $N^{\circ} 46$, pp. 41-72.

Cunill Grau, Nuria (2011). Responsabilización y exigibilidad de los derechos en salud ¿Qué se puede aprender de la reforma sanitaria chilena? Santiago, Chile, Mimeo.

Cunill Grau, Nuria; Fernández, Margarita y Vergara, Marcos (2011). Gobernanza Sistémica para un Enfoque de Derechos en Salud. Un análisis a partir del caso chileno. Salud Colectiva 7 (1), pp. 21-33.

División de Organizaciones Sociales (2009). Experiencias Exitosas de Cuentas Públicas Participativas, Santiago: División de Organizaciones Sociales. Ministerio Secretaría General de Gobierno, Santiago, Chile.

Dowling, Bernard; Sheaff, Rod y Pickard, Susan (2008). Governance structures and accountability in primary care. Public Money \& Management, 28 (4), pp. 215-222.

Drago, Marcelo (2006). La reforma al sistema de salud chileno desde la perspectiva de los derechos humanos, Santiago: CEPAL. Serie Políticas Sociales. № 121.

Dworkin, Ronald (2002). Los derechos en serio. Barcelona, España. Ariel.

Erazo, Alvaro y Titelman, Daniel (2008). Cobertura y garantías en salud. En: Erazo, Ximena; Abramovich, Víctor y Orbe, Jorge (eds.). Políticas pública para un Estado social de derechos. El paradigma de los derechos universales. Santiago de Chile: LOM ediciones.

Fung, Archon (2003). Survey Article. Recipes for Public Spheres: Eight Institutional Design Choices and their Consequences. The Journal of Political Philosophy, 11 (3), pp. 338-67.
Fung, Archon y Wright, Erik Olin (2003). En torno al Gobierno Participativo con Poder de Decisión. En: Fung, Archon, Wright, Erik Olin (edits.). Democracia en profundidad. Bogotá: Universidad Nacional de Colombia.

Gherardi, Natalia (2009). La administración pública y el acceso a la justicia: una oportunidad para la materialización de los derechos sociales. En: Abramovich $V$ y Pautassi L (comps.) La revisión judicial de las políticas sociales. Buenos Aires: Ediciones del Puerto; pp. 243-77.

Goldsmith, Stepher y Eggers, William D. (2006). Governar em rede. O novo formato do setor publico, Brasilia: Ed. UNESP.

Herreño Hernández, Ángel Libardo (2008). ¿Todo o nada? Principio de integralidad y derechos sociales. Bogotá: Publicaciones ILSA.

Hufty, Marc (2010). Gobernanza en salud pública: hacia un marco analítico. Revista de Salud Pública, vol. 12, supl. 1 Bogotá. En: http://doi.org/10.1590/ SO124-0064010000700004 (fecha de consulta: 2/02/2012).

Infante, Antonio y Paraje, Guillermo (2010). Reforma de Salud: Garantías Exigibles como Derecho Ciudadano. En: Larrañaga, Osvaldo y Contreras, Dante (editores) Las nuevas políticas de protección social en Chile. Santiago de Chile: Editorial Uqbar.

Ministerio de Salud (2004a) Ley $\mathbf{N}^{\circ}$ 19.937. Modifica el D.L. № 2.763, de 1979, con la finalidad de establecer una nueva concepción de la autoridad sanitaria, distintas modalidades de gestión y fortalecer la participación ciudadana. Diario Oficial, 24 de febrero de 2004, Chile.

Ministerio de Salud (2004b). Ley $\mathbf{N}^{\circ} \mathbf{1 9 . 9 6 6 .}$ FONASA. Establece un régimen de 
garantías en salud. Diario Oficial, 3 de septiembre de 2004, Chile.

Ministerio de Salud (2004c). Ley $\mathbf{N}^{\circ}$ 18.469. Regula el ejercicio del derecho constitucional a la protección de la salud y crea un régimen de prestaciones en salud. Diario Oficial, 3 de Septiembre de 2004, Chile.

Ministerio de Salud (2005a). Decreto 170. Aprueba Garantías Explícitas en Salud del Régimen General de Garantías en Salud. Diario Oficial, 28 de enero de 2005, Chile.

Ministerio de Salud (2005b). Reglamento 136. Reglamento Orgánico del Ministerio de Salud. Diario Oficial, 21 de Abril del 2005, Chile.

Ministerio de Salud (2005c). Reglamento 128. Reglamento sobre el sistema de acreditación a que se refieren los artículos 16 y siguientes de la Ley № 19.664. Diario Oficial, 5 de Mayo del 2005, Chile.

Ministerio de Salud (2006). DFL 1. Establece Estructuras y Funciones del Ministerio de Salud y servicios desconcentrados. Diario Oficial, 24 de Abril de 2006, Chile.

Ministerio de Salud (2007). Reglamento 15. Ministerio de Salud. Reglamento del Sistema de Acreditación para los prestadores institucionales de Salud. Diario Oficial, 3 de marzo de 2007, Chile

Ministerio de Salud (2008a). "Compromisos de Gestión y sus Orientaciones". Departamento Control de Gestión. Santiago. Chile.

Ministerio de Salud (2008b). Plan de Salud Pública y Promoción de Salud desafíos en el contexto del Sistema de Protección Social. Dra Helia Molina Jefa División Políticas Públicas Saludables y Promoción de la Salud. Taller de Establecimientos Promotores la Salud, 7 y 8 de abril. Santiago, Chile, pp 33.
Ministerio de Salud (2009). Participación Social en Salud: Reflexiones y Herramientas para la Acción desde Chile. Seminario Internacional "Gestión Pública y Participación Social", 23 y 25 de marzo de 2009, Santiago, Chile. Proyecto Eurosocial Salud. Proyecto Regional para la Cohesión Social en Salud en América Latina, pp 325.

Ministerio de Salud (2010). Decreto 1. Aprueba Garantías Explícitas en Salud del Régimen General de Garantías en Salud. Diario Oficial, 27 de febrero de 2010, Chile.

Ministerio Secretaría General de Gobierno (2007). Agenda Pro Participación Ciudadana. Gobierno de Chile. Santiago, Atenas, pp 228.

Ministerio Secretaría General de Gobierno (2011). Ley 20.500. Sobre Asociaciones y Participación Ciudadana en la Gestión Pública. Diario Oficial № 39.838, Santiago. Chile.

Notti, Francesco (2008). El enfoque de derechos humanos en el desarrollo. En: Erazo, Ximena; Abramovich, Víctor y Orbe, Jorge (eds.), Políticas pública para un Estado social de derechos. El paradigma de los derechos universales. Santiago de Chile: LOM ediciones.

Osborne, Stephen P. (2010). Introduction. The (New) Public Governance: a suitable case for treatment? En: Osborne, Stephen P. (editor), The New Public Governance? Emerging perspectives on the theory and practice of public gobernance, London and NY: Routledge, Taylor \& Francis Group.

Pautassi, Laura (2010). El enfoque de derechos y la inclusión social. Una oportunidad para las políticas públicas. En: Pautassi, Laura (org.), Perspectiva de derechos, políticas públicas e inclusión social. Debates actuales en la Argentina. Buenos Aires, Argentina. Biblos. 
Pautassi, Laura (2007). El cuidado como cuestión social desde un enfoque de derechos. Santiago: CEPAL. Serie Mujer y Desarrollo. $N^{\circ} 87$, pp 50.

Pozo Carlos y Cancino, Anselmo. (2010). Los Presupuestos Participativos: una herramienta de deliberación democrática para la construcción de ciudadanía en Salud: el caso de tres Servicios de Salud de la Región Metropolitana, Tesis para optar al grado de Magister en Gerencia Social y Salud. Flacso Chile.

Santos, Boaventura de Sousa y Avritzer, Leonardo (2004). Introducción: para ampliar el canon democrático. En: Santos, Boaventura de Sousa (coord.), Democratizar la democracia. México: Fondo de Cultura Económica.

Shankardass, Ketan; Solar, Orielle; Murphy, Kelly; Greaves, Lorraine y O'Campo, Patricia (2012). A scoping review of intersectoral action for health equity involving governments. International Journal of Public Health $\mathrm{N}^{\circ} 57$, pp. 25-33.

United Nations (2002). Draft Guidelines: a Human Rights Approach to Poverty Reduction Strategies.

Walters, Lawrence C.; Aydelotte, James; Miller, Jessica (2000). Putting More Public in Policy Analysis. Public Administration Review, 60 (4).

\section{Entrevistas}

Entrevista Grupal 1. Dirigentes del Servicio de Salud Occidente, 23 septiembre de 2010.

Entrevista Grupal 2. Dirigentes del Servicio de Salud Sur Oriente, 25 septiembre de 2010.

Entrevista Grupal 3. Dirigentes del Servicio de Salud O'Higgins, 15 octubre de 2010.

Entrevista Grupal 4. Dirigentes del Servicio de Salud Ñuble, 10 noviembre de 2010.
Entrevista Grupal 5. Dirigentes del Servicio de Salud Talcahuano, 15 noviembre de 2010.

Entrevistado 1. Director del Servicio de Salud Metropolitano Occidente. Región Metropolitana-Santiago,23 de noviembre de 2009.

Entrevistado 2. Director del Servicio de Salud Metropolitano Sur Oriente. Región Metropolitana-Santiago, 23 de noviembre de 2009.

Entrevistado 3. Director Hospital B. Servicio de Salud Metropolitano Occidente. San José de Maipo, 26 de noviembre de 2009.

Entrevistado 4. Jefa de División de Salud Municipalidad de La Florida. Servicio de Salud Metropolitano Sur Oriente. Santiago, 27 de noviembre de 2009.

Entrevistado 5. Dirección de Salud Municipalidad Isla de Maipo. Servicio de Salud Metropolitano Occidente. Isla de Maipo, 2 de diciembre de 2009.

Entrevistado 6. Director de Salud Ignacio Domeyko. Servicio de Salud Metropolitano Occidente. Santiago, 3 de diciembre de 2009.

Entrevistado 7. Director Servicio de Salud O'Higgins. Región Libertador Bernardo O'Higgins-Rancagua, 7 de diciembre de 2009.

Entrevistado 8. Subdirectora Servicio de Salud O’Higgins. Región Libertador Bernardo O'Higgins-Rancagua, 7 de diciembre de 2009.

Entrevistado 9. Director del Hospital Base Rancagua. Región Libertador Bernardo O'Higgins-Rancagua, 8 de diciembre de 2009.

Entrevistado 10. Directora del CESFAM № 3. Rancagua. Municipalidad de Rancagua. Región Libertador Bernardo O'Higgins-Rancagua, 8 de diciembre de 2009. 
Entrevistado 11. Directora del Hospital Pichilemu. Servicio de Salud O'Higgins. Región Libertador Bernardo O'HigginsPichilemu, 9 de diciembre de 2009.

Entrevistado 12. Subdirector del Servicio de Salud Ñuble. Región del Bìo-Bìo-Chillán,11 de diciembre de 2009.

Entrevistado 13. Director del Hospital Base A. Servicio de Salud Ñuble, Región del Bío-Bío-Chillán, 11 de diciembre de 2009.

Entrevistado 14. Directora de Salud Municipalidad de San Nicolas. Servicio de Salud Ñuble. Región del Bío-Bío-Chillán, 11 de diciembre de 2009.

Entrevistado 15. Directora del CESFAM Violeta Parra. Chillán, 14 diciembre de 2009

Entrevistado 16. Director del Servicio de Salud Ñuble. Chillán,14 de diciembre de 2009.

Entrevistado 17. Subdirector de Redes Asistenciales Servicio de Salud Talcahuano. Talcahuano, 15 de diciembre de 2009.

Entrevistado 18. Director del Servicio de Salud Talcahuano. Talcahuano, 15 de diciembre de 2009.

Entrevistado 19. Director del Hospital Base A. Servicio de Salud Talcahuano. Talcahuano, 15 de diciembre de 2009.
Entrevistado 20. Superintendente de la Superintendencia de Salud. Santiago, 24 de noviembre de 2009.

Entrevistado 21. Director Departamento de Salud, Municipalidad de Talcahuano, Servicio de Salud de Talcahuano, 6 de diciembre de 2009.

Entrevistado 22. Director Departamento de Salud, Municipalidad de Chillán, Servicio de Salud Ñuble,10 de diciembre de 2009.

Entrevistado 23. Director Departamento de Salud, Municipalidad de Litueche, Servicio de Salud O"Higgins, 10 diciembre de 2009.

Entrevistado 24. Directora Departamento de Salud, Municipalidad de Rancagua, Servicio de Salud O"Higgins, 8 diciembre de 2009.

Entrevistado 25. Director Departamento de Salud, Municipalidad de Tomé, Servicio de Salud Nuble. 6 diciembre de 2009.

Entrevistado 26. Directora área salud Corporación Municipal de Salud de Quinta Normal, Servicio de Salud Occidente, 10 noviembre de 2009

Entrevistado 27. Directora Salud y Educación, Municipalidad de La Pintana, Servicio de Salud Occidente 14 diciembre de 2009. 